\title{
IN VITRO ANTIANGIOGENESIS ACTIVITY OF STANDARDIZED EXTRACTS OF Piper sarmentosum Roxb
}

\author{
Khalid Hussain, Zhari Ismail, Amirin Sadikun, Pazilah Ibrahim, Amin Malik \\ School of Pharmaceutical Science, Universiti Sains Malaysia, Minden Campus Pulau Penang- \\ 11800. Malaysia. Phone and Fax 006046563443. \\ email: hussain_761@yahoo.com
}

\begin{abstract}
This study was undertaken to investigate the antiangiogenesis activity of standardized extracts/fractions of the leaf of Piper sarmentosum, using rat aorta model. The pulverized leaf was extracted sequentially and methanol extract was further fractionated with hexane, chloroform and ethylacetate. Both extracts and fractions were standardized by reverse phase HPLC with UV detection at $260 \mathrm{~nm}$, using two markers, sarmentine and sarmentosine. Chloroform and methanol extracts have exhibited antiangiogenesis activity of $100 \%$ and $20 \%$ respectively. Antiangiogenesis activity of hexane and chloroform fractions was found to be $10 \%$ and $90 \%$ respectively, while ethylacetate fraction was found to be inactive. The analysis of most active extract and fraction has exhibited different profile by HPLC on the basis of amides. This study indicates that chloroform extract and fraction have promising antiangiogenesis activity and have potential for diseases involving angiogenesis.
\end{abstract}

Keywords : antiangiogenesis activity, Piper sarmentosum Roxb. 


\section{REFERENCES}

1. Goto, F., Goto, K., Weindel, K., Folkman, J., 1993, Synergistic effects of vascular endothelial growth-factor and basic fibroblast growth factor on the proliferation and cord formation of bovine capillary endothelial cells within collagen gels, Lab. Inves., 69: 508-17.

2. Prior, B. M., Yang, H. T., Terjung, R. L., 2004, What makes vessels grow with exercise training?, J. App. Physiol., 97: 1119-28.

3. Haas, T. L., Milkiewicz, M., Davis, S. J., Zhou, A. L., Egginton, S., Brown, M. D., Madri, J. A., Hudlicka, O., 2000, Matrix metalloproteinase activity is required for activity-induced angiogenesis in rat skeletal muscle, Am. J. Physiol. Heart Circ. Physiol., 279: H1540-H1547.

4. Thurston, G., 2003, Role of Angiopoietins and Tie receptor tyrosine kinases in angiogenesis and lymphangiogenesis, Cell Tissue Res., 314(1): 61-8.

5. Lobov, I. B., Renard, R. A., Papadopoulos, N., Gale, N. W., Thurston, G., Yancopoulos, G. D., Wiegand, S. J., 2007, Delta-like ligand 4 (D114) is induced by VEGF as a negative regulator of angiogenic sprouting, Proc. Natl. Acad. Sci. USA., 104(9): 3219-24.

6. Toong, V. Y., Wong, B. L., 1989, Phytochemistry of Medicinal Plants: Piper sarmentosum. Proceedings: Traditional Medicine, Kulala Lumpur, 1998. Institute of Advance Studies, University of Malaya, Kuala Lumpur, 280.

7. Masuda, T., Ingumi, A., Yamada, Y., Padolina, W. G., Kikuzaki, H., Nakatani, N., 1995, Antimicrobial phenyl propanoids from Piper sarmentosum, Phytochemistry, 39(3): 731-733.

8. Stoehr, J. R., Xiao, P. G., Bauer, R., 1999, Isobutylamides and a new (methylbutyl) amide from Piper sarmentosum, Planta Med., 65(2): 175-177.

9. Thitima, R., Puttan, S., Kanchanawadee, S., Chanika, W., Phongpan, R., Phaopong, W., Apichart, S., 2004, Chemical constituents and bioactivity of Piper sarmentosum, J. Ethnopharmacol, 93: 173-176.

10. Tutiwachwuttikul, P., Phansa, P., Pootaeng, OnY, Tylor, W. C., 2006, Chemical constituents of the roots of Piper sarmentosum, Chem. Pharm. Bull., 54(2): 149-51.

11. Hock-Hin, Y., Pek-Funn, M. W., 1993, Food value of lesser utilized tropical plants, Food. Chem., 3: 239-241.

12. Vimala, S., Mohd. IIham, A., Abdul Rsshid, A., Rohana, S., 2003, Natural antioxidants: Piper sarmentosum (Kadok) and Morinda elliptica (Mengkudu), M. J. Nutri., 9(1): 41-51.

13. Anchana, C., Aphiwat, T., Nuansri, R., 2005, Screening of antioxidant activity and antioxidant compounds of some edible plants of Thailand, Food Chem., 92: 491-497.

14. Ridtitid, W., Rattanaprom, W., Thaina, P., Chittrakarn, S., Sunbhanich, M., 1998, Neuromuscular blocking activity of methanolic extract of Piper sarmentosum leaves in the rat phrenic nerve-hemidiaphragm preparation, $J$. Ethnopharmacol,61(2): 135-42.

15. Peungvicha, P., Thirawarapan, S. S., Temsiririrkkul, R., Watanabe, H., Kumar Prasain, J., Kadota, S., 1998, Hypoglycemic effect of the water extract of Piper sarmentosum in rats, $J$. Ethnopharmacol, 60(1): 27-32.

16. Choochote, W., Chaithong, U., Kamsuk, K., Rattanachanpichai, E., Jitpakdi, A., Tippawangkosol, P., Chaiyasit, D., Champakaew, D., Tuetun, B., Pitasawat, B., 2006, Adulticidal activity against Stegomyia aegypti (Diptera: Culicidae) of three Piper spp, Rev. Inst. Med. Trop. Sao Paulo, 48(1): 33-7.

17. Sawangjaroen, N., Sawangjaroen, K., Poonpanang, P., 2004, Effects of Piper longum fruit, Piper sarmentosum root and Quercus infectoria nut gall on caecal amoebiasis in mice, J. Ethnopharmacol, 91(2-3): 357-60.

18. Rukachaisirikul, T., Siriwattanakit, P., Sukcharoenphol, K., Wongvein C., Ruttanaweang, P., Wongwattanavuch, P., Suksamrarn, A., 2004, Chemical constituents and bioactivity of Piper sarmentosum, J. Ethnopharmacol, 93(23): 173-6.

19. Brown, K. J., Maynes, S. F., Bezos, A., Maguire D. J., Ford M. D., Parish, C. R., 1996, A Novel In vitro Assay for Human Angiogenesis, Laboratory Investigation, 75: 539-555. 
